\title{
Influence of arthritis-related protein (BBF01) on infectivity of Borrelia burgdorferi B31
}

\author{
Denise Imai, Kevin Holden, Eric M Velazquez, Sunlian Feng, Emir Hodzic and Stephen W Barthold*
}

\begin{abstract}
Background: Lyme borreliosis, caused by tick-borne Borrelia burgdorferi, is a multi-phasic, multi-system disease in humans. Similar to humans, $\mathrm{C} 3 \mathrm{H}$ mice develop arthritis and carditis, with resolution and periodic bouts of recurrence over the course of persistent infection. Borrelia burgdorferi arthritis-related protein (Arp/BBF01), a highly conserved protein among B. burgdorferi s.s. isolates, has been shown to be antigenic in humans with Lyme borreliosis, and a target for antibody-mediated disease resolution in the mouse model.

Results: A mutant strain of B. burgdorferi s.s. deficient of the arp gene and a complemented version of that mutant were created and examined for phenotypic effects in mice compared to wild-type B. burgdorferi. Deletion of arp did not abolish infectivity, but did result in a higher infectious dose compared to wild-type B. burgdorferi, which was restored by complementation. Spirochete burdens in tissues of $\mathrm{C} 3 \mathrm{H}$-scid mice were lower when infected with the arp mutant, compared to wild-type, but arthritis was equally severe. Spirochete burdens were also lower in $\mathrm{C} 3 \mathrm{H}$ mice infected with the arp mutant, but disease was markedly reduced. Ticks that fed upon infected $\mathrm{C} 3 \mathrm{H}$ mice were able to acquire infection with both wild-type and arp mutant spirochetes. Arp mutant spirochetes were marginally able to be transmitted to naïve hosts by infected ticks.

Conclusion: These results indicated that deletion of BBF01/arp did not abrogate, but diminished infectivity and limited spirochete burdens in tissues of both immunocompetent and immunodeficient hosts, and attenuated, but did not abolish the ability of ticks to acquire or transmit infection.
\end{abstract}

Keywords: Lyme borreliosis, Arthritis related protein, BBF01

\section{Background}

Lyme disease, caused by tick-borne Borrelia burgdorferi, is a multi-systemic and multi-phasic disease in humans, which includes pauciarticular arthritis in up to $60 \%$ of untreated patients [1,2]. In the absence of antibiotic treatment, arthritis and other lesions undergo resolution with variable bouts of recurrence over the course of months to years of persistent infection [3]. Laboratory mice develop arthritis and carditis that follow a similar multi-phasic course as humans, with resolution and periodic bouts of recurrence over the course of persistent infection [4]. The mouse model has implicated the humoral immune response as a critical factor in arthritis and carditis resolution. Infection of T-cell deficient (Tcr $\alpha / \beta$ null, Tcr $\gamma / \delta$-null), but not B-cell deficient (Igh6-null) or severe combined immunodeficient (SCID) or Rag1-

\footnotetext{
* Correspondence: swbarthold@ucdavis.edu

Center for Comparative Medicine, Schools of Veterinary Medicine and

Medicine, University of California, One Shields Avenue, Davis, CA 95616, USA
}

null mice follows a course of resolution that is similar to fully immunocompetent mice [5], and passive transfer of serum from actively infected immunocompetent mice that have undergone disease resolution (immune serum) into infected SCID mice results in complete resolution of arthritis and carditis, but not clearance of infection [6-8].

Identification of the B. burgdorferi antigens targeted by antibodies that mediate disease resolution is complicated by the fact that $B$. burgdorferi grown in culture medium does not reflect the antigenic profile of spirochetes during mammalian infection $[9,10]$. As a means to identify vulnerable antigenic targets that are expressed in the mammalian host that are responsible for antibodymediated disease resolution, immune serum from actively infected mice has been used to probe $B$. burgdorferi genomic expression libraries or outer membrane extracts. These efforts revealed arthritis-related protein (BBF01/Arp) as well as decorin binding protein 
A (DbpA), among other antigens expressed during infection [8,11-13]. Antiserum generated in mice hyperimmunized with non-lipidated recombinant Arp or DbpA induced arthritis and carditis resolution, but did not eliminate infection, when passively transferred to actively infected SCID mice [8,12]. Immunization with DbpA was found to induce protective immunity against cultured spirochetes [11,14], but not tick-borne spirochetes [15], whereas Arp immunization was ineffective at eliciting protective immunity against cultured spirochetes [16]. Outer surface protein $\mathrm{C}(\mathrm{OspC})$, another immunogenic protein expressed during infection, has also been shown to be vulnerable to passively transferred OspC antibody in SCID mice, but is down-regulated in response to specific antibody, thereby avoiding immune clearance in immunocompetent mice $[17,18]$.

Based upon the presence of a leader sequence, arp encodes a putative lipoprotein that is expressed in ticks and throughout the course of mammalian infection and has been shown to stimulate the production of antibody in both experimentally infected mice and naturally infected humans [13,16,19-21]. Genomic comparison among several B. burgdorferi sensu stricto (s.s.) strains reveals highly conserved BBF01/arp sequences (95-100\% identity from GenBank Blast). Curiously, the genomes of other B. burgdorferi sensu lato strains that are available in GenBank, such as B. afzelii and B. garinii, do not appear to have an arp homolog. In contrast to arp conservation in B. burgdorferi s.s. strains, $\operatorname{dbp} A$ and $o s p C$, which also encode immunogenic antigens that are expressed during infection [19,21-23], have considerable variation (81-85\% identity) among the same $B$. burgdorferi s.s. strains (GenBank). As noted, both Arp and DbpA stimulate an arthritis-resolving immune response [8], and DbpA and OspC elicit protective immune responses against challenge $[11,14,24]$. It is therefore curious that Arp has such a conserved sequence among B. burgdorferi s.s. strains, when it is so obviously subjected to immune selection pressure. The present study explored the biological behavior of $B$. burgdorferi devoid of, or complemented with, Arp. Arp was found to be non-essential for infectivity, but it influenced infectious dose, spirochete burdens in tissues, arthritis severity, and tick infection kinetics, underscoring its biological significance.

\section{Results}

Seven B. burgdorferi B31-arp deletion mutants ( $\Delta$ arp) were created, and found to grow equally well in BSKII medium as B31 (wild-type) spirochetes. The $7 \Delta$ arp mutants were initially tested for infectivity in infant ICR mice, which serve as an inexpensive system for titrating infectivity [5]. All seven mutants were determined to be flagellin $B$ (flaB) DNA-positive and arp DNA-negative by polymerase chain reaction (PCR), following growth selection in streptomycin. Four 2-day-old mice were inoculated with $10^{6}$ of each $\Delta$ arp mutant or wild-type spirochetes, and sub-inoculation site and urinary bladder were cultured to determine infectivity and ability to disseminate at 7 and 21 days after inoculation. All were infectious, and all disseminated to the urinary bladder. Spirochetes cultured from the inoculation site and urinary bladder were tested by PCR for presence of flaB and arp. Urinary bladder isolates from mice that were flaBpositive and arp-negative were selected for further analysis and confirmed to be arp-null. Upon subsequent inoculation of infant ICR mice with wild-type or each of the seven $\Delta$ arp mutants, arthritis was of equivalent severity as mice infected with B31 among all groups of mice, indicating that $B$. burgdorferi devoid of arp were not only infectious, but also equally pathogenic as wildtype $B$. burgdorferi in susceptible infant mice. One arp isolate $(\Delta \mathrm{arp} 3)$ was selected for further analysis.

The median infectious dose $\left(\mathrm{ID}_{50}\right)$ of $\Delta$ arp3 was compared to wild-type and to $\Delta$ arp3 complemented with the plasmid lp28-1G containing arp ( $\Delta$ arp3+lp28-1G). Groups of 4 infant ICR mice were inoculated subdermally with $10^{1}, 10^{2}, 10^{3}, 10^{4}$, or $10^{5}$ spirochetes. Mice were necropsied at 2 weeks, and sub-inoculation site and urinary bladder were cultured (Table 1 ). The $\mathrm{ID}_{50}$ of wild-type was $5 \times 10^{3}$ spirochetes, whereas the $\mathrm{ID}_{50}$ of $\Delta$ arp3 was $8 \times 10^{4}$ spirochetes. Relative infectivity could be restored by complementation of the $\Delta$ arp3 mutant with 1 28-1G, resulting in an ID $_{50}$ identical to wild-type. Subsequent experiments in $\mathrm{C} 3 \mathrm{H}$ and $\mathrm{C} 3 \mathrm{H}$-scid mice therefore used an infectious dose of $10^{5}$ or greater spirochetes.

Four C3H-scid mice were each inoculated with $10^{6}$ wild-type and five $\mathrm{C} 3 \mathrm{H}$-scid mice were each inoculated with $10^{6} \Delta$ arp3 spirochetes, and then necropsied at 60 days of infection to compare the full range of pathogenicity of each inoculum, unencumbered by acquired immunity. All inoculation sites and urinary bladders were culture-positive in both groups. Spirochetes were

\begin{tabular}{|c|c|c|c|}
\hline Inoculum dose & $\Delta$ arp3 & $\Delta \operatorname{arp3}+\operatorname{Ip28-1G}$ & wild-type \\
\hline$\overline{10^{1}}$ & $0 / 4^{*}$ & $0 / 4$ & $0 / 4$ \\
\hline $10^{2}$ & $0 / 4$ & $0 / 4$ & $0 / 4$ \\
\hline $10^{3}$ & $0 / 4$ & $0 / 4$ & $0 / 4$ \\
\hline $10^{4}$ & $1 / 4$ & $4 / 4$ & $4 / 4$ \\
\hline $10^{5}$ & $2 / 4$ & $4 / 4$ & $4 / 4$ \\
\hline
\end{tabular}

* number of positive mice/number of mice tested. 
isolated from blood of 4/4 wild-type inoculated mice, whereas only $2 / 4$ (one sample not collected) $\Delta$ arp3 inoculated mice were bacteremic. All mice in both groups had severe (mean arthritis score $3.0 \pm 0 \mathrm{SD}$ ) arthritis in tibiotarsal joints, as well as arthritis in both knees, and all mice had carditis. Despite equally severe disease, spirochete burdens in sub-inoculation, heart base, and tibiotarsal tissues, based upon flaB quantitative PCR (Q$\mathrm{PCR})$, were significantly lower $(\mathrm{P} \leq 0.05)$ in $\Delta$ arp3 infected $\mathrm{C} 3 \mathrm{H}$-scid mice compared to wild-type infected mice (Figure 1). Spirochete burdens were also lower in ventricular muscle and quadriceps muscle, but differences were not statistically significant.

A confirmatory experiment was performed in which 5 $\mathrm{C} 3 \mathrm{H}$-scid mice were each inoculated with $10^{6}$ wild-type and $5 \mathrm{C} 3 \mathrm{H}$-scid mice were each inoculated with $10^{6}$ $\Delta$ arp3 spirochetes, and necropsied on day 28 after inoculation. Inoculation sites and urinary bladders in all mice from both groups were culture-positive, and all mice in both groups were bacteremic. Arthritis severity scores were equivalent in both groups (mean $2.8 \pm 0.4$ SD wild-type vs. mean $2.4 \pm 0.5$ SD $\Delta$ arp3). Significantly lower flaB Q-PCR spirochete burdens $(P \leq 0.05)$ were found in tissues of $\Delta$ arp3 infected $\mathrm{C} 3 \mathrm{H}$-scid mice compared to wild-type infected $\mathrm{C} 3 \mathrm{H}$-scid mice, including inoculation site $(320 \pm 486$ SD vs $249,660 \pm 187,097$ SD), heart base $(983 \pm 1,353$ SD vs $514,400 \pm 171,404$ SD), ventricular muscle $(9,656 \pm 5,911 S D$ vs $432,260 \pm$ 374,173 SD), and tibiotarsus (201 \pm 187 SD vs $163,520 \pm$ 89,127 SD). Spirochete burdens were also reduced in quadriceps muscle $(3,730 \pm 1,412 \mathrm{SD}$ vs $58,640 \pm 74,839$ $\mathrm{SD})$, but differences were not statistically significant $(P=0.07)$.

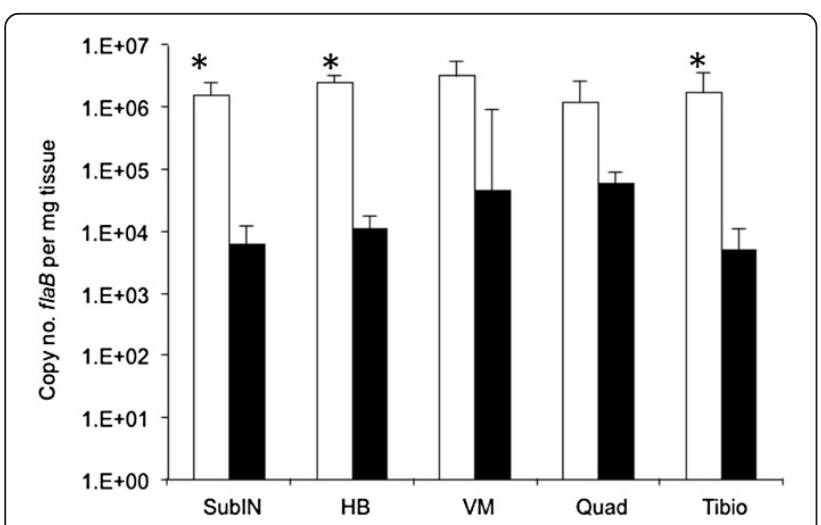

Figure 1 Borrelia burgdorferi flaB DNA copies per mg tissue weight (means \pm standard deviations) in subinoculation site (subIN), heart base (HB), ventricular muscle (VM), quadriceps muscle (Quad) and tibiotarsus (Tibio) from $4 \mathrm{C} 3 \mathrm{H}$-scid mice inoculated with wild-type (white bars) compared to $5 \mathrm{C} 3 \mathrm{H}$-scid mice inoculated with arp null $\Delta \operatorname{arp} 3 B$. burgdorferi (black bars). $(*, P \leq 0.05)$
Next, groups of 5 immunocompetent $\mathrm{C} 3 \mathrm{H}$ mice were inoculated with $10^{5}$ wild-type or $\Delta$ arp3 spirochetes, and then necropsied on days 14, 28 and 42. Tissues were examined for arthritis and carditis, and flaB Q-PCR was performed on sub-inoculation site, heart base, ventricular muscle, tibiotarsus and quadriceps muscle. Inoculation sites of all mice were culture-positive at each interval tested, but none of the urinary bladders of mice inoculated with $\Delta$ arp3 were culture-positive at 14 days, suggesting delayed dissemination, or reduced sensitivity due to lower tissue burdens (Table 2). Compared to inoculation site, urinary bladders were less consistently culture-positive in both groups of mice, underscoring the greater accuracy of PCR for assessing dissemination and tissue burdens (Table 3). At 14 days, $1 / 5$ wild-type inoculated mice had $1+$ inflammation of the tibiotarsus and 5/ 5 had carditis, whereas none of the $\Delta$ arp3 inoculated mice had inflammatory lesions in joints or heart at this interval. At 28 days, 5/5 wild-type inoculated mice had both arthritis (1.6 \pm 0.5 SD severity) and carditis, whereas only $1 / 5 \Delta$ arp3 inoculated mice had carditis and none had arthritis. At 42 days, 3/3 wild-type inoculated mice continued to have arthritis $(1.5 \pm 0.5 \mathrm{SD})$ and carditis, and $1 / 5 \Delta$ arp3 mice had arthritis ( $1+$ severity) and $2 / 5$ had carditis. PCR-positive tissue samples at all intervals indicated that wild-type infected mice had higher spirochete burdens in tissues compared to $\Delta$ arp3 infected mice (Figure 2). At day 14, most tissues from wild-type inoculated mice were PCR-positive, whereas very few tissues from $\triangle$ arp3 inoculated mice were PCR-positive (Table 3). The rate of PCR-positive tissues increased in the $\Delta$ arp3 inoculated mice on days 28 and 42 to rates similar to wild-type infected mice, but flaB DNA copy numbers were consistently lower.

A confirmatory experiment was performed in which groups of $4 \mathrm{C} 3 \mathrm{H}$ mice were inoculated with $10^{6}$ wildtype or $\Delta$ arp3 spirochetes, and then necropsied on day 28 to verify the difference in tissue spirochete burdens in heart base, ventricular muscle, quadriceps muscle, and tibiotarsal tissue. Tissues were not collected for histopathology. In wild-type infected mice, 4/4 inoculation sites and $3 / 4$ urinary bladders were culture-positive, and $3 / 3$ inoculation sites (one sample contaminated) and $0 / 4$ urinary bladders were culture-positive in $\Delta$ arp3 infected mice. Spirochete burdens were significantly lower $(P \leq 0.05)$ in tissues of $\Delta$ arp3 infected mice compared to wild-type infected mice, including subinoculation site $(139 \pm 266 \mathrm{SD}$ vs. $1,761 \pm 1,682 \mathrm{SD})$, heart base ( $45 \pm 54 \mathrm{SD}$ vs. 2,333 $\pm 1,400 \mathrm{SD})$, ventricular muscle ( $28 \pm 26$ SD vs $448 \pm 276$ SD), and quadriceps muscle $(15 \pm 23$ SD vs $367+291$ SD). Spirochete burdens were also lower in tibiotarsus tissue of $\Delta$ arp3 infected mice $(13 \pm 11$ SD vs $16,171 \pm 29,765$ SD), but differences were not statistically different $(P=0.16)$. 
Table 2 Outcome of infection of $\mathrm{C} 3 \mathrm{H}$ mice with wild-type vs. arp null ( $\Delta$ arp3) Borrelia burgdorferi at intervals (days) after inoculation

\begin{tabular}{|c|c|c|c|c|c|c|}
\hline \multirow[b]{2}{*}{ Day } & \multicolumn{3}{|c|}{ Culture } & \multicolumn{3}{|c|}{ Inflammation } \\
\hline & Inoculum & Inoc. site & Urinary bladder & Tibiotarsus & Knee & Heart \\
\hline \multirow[t]{2}{*}{14} & wild-type & $5 / 5^{*}$ & $3 / 5$ & $1 / 5$ & $0 / 5$ & $5 / 5$ \\
\hline & $\Delta$ arp3 & $5 / 5$ & $0 / 5$ & $0 / 5$ & $0 / 5$ & $0 / 5$ \\
\hline \multirow[t]{2}{*}{28} & wild-type & $5 / 5$ & $4 / 5$ & $5 / 5$ & $4 / 5$ & $5 / 5$ \\
\hline & $\Delta$ arp3 & $5 / 5$ & $2 / 4^{* *}$ & $0 / 5$ & $0 / 5$ & $1 / 5$ \\
\hline \multirow[t]{2}{*}{42} & wild-type & $3 / 3$ & $2 / 3$ & $3 / 3$ & $0 / 3$ & $1 / 3$ \\
\hline & $\Delta$ arp3 & $4 / 4^{* *}$ & $5 / 5$ & $1 / 5$ & $0 / 5$ & $2 / 5$ \\
\hline
\end{tabular}

* number positive/number tested.

** one culture sample contaminated.

Based upon these observations, it was determined that both $\mathrm{C} 3 \mathrm{H}$-scid mice as well as $\mathrm{C} 3 \mathrm{H}$ mice infected with $\Delta$ arp3 had lower spirochete burdens in tissues.

Sera from $\mathrm{C} 3 \mathrm{H}$ mice that were confirmed to be culture-positive at 60 days of infection with wild-type or $\Delta$ arp3 spirochetes were determined to be appropriately sero-reactive against recombinant Arp antigen (Arp seropositive or seronegative, respectively). Serum antibody titers from $\Delta$ arp3 infected mice were equivalent to antibody titers in mice infected with wild-type infected mice when tested against $B$. burgdorferi lysate antigen $(\geq 1: 24,300)$, and antibody titers to recombinant Arp antigen were verified to be either negative $(\Delta \mathrm{arp} 3)$ or positive $(\Delta \operatorname{arp} 3+\operatorname{lp} 28-1 G)$, with titers equivalent to Arp titers in wild-type immune sera $(1: 2,700)$.

Larval ticks were fed upon the before-mentioned wildtype or $\Delta$ arp3 infected $\mathrm{C} 3 \mathrm{H}$ mice 3 days before necropsy at day 42 . Replete ticks were allowed to molt and harden into nymphs, and then tested by Q-PCR for flaB and arp DNA. Among ticks that fed upon wild-type infected mice, 30/30 were PCR positive for both flaB and arp, with 53,950 mean $\pm 84,668$ SD flaB copy numbers per tick. In contrast, $40 / 50$ ticks that fed upon $\Delta$ arp3 infected mice were $f l a B$-positive, with significantly lower spirochete loads per positive tick $(1,384$ mean $\pm 1,780$

Table 3 Rate of PCR (flaB DNA) positivity of subinoculation site, heart base, ventricular muscle, quadriceps muscle and tibiotarsus tissue from $\mathrm{C} 3 \mathrm{H}$ mice at intervals (days) after inoculation with wild-type vs. arp null ( $\Delta$ arp3) Borrelia burgdorferi

\begin{tabular}{lcccccc}
\hline Day & Inoculum & SubIN & Hrt base & Vent M & Quad M & Tibiotarsus \\
\hline 14 & wild-type & $4 / 5^{*}$ & $5 / 5$ & $5 / 5$ & $4 / 5$ & $4 / 5$ \\
& Darp3 & $2 / 5$ & $1 / 5$ & $0 / 5$ & $0 / 5$ & $0 / 5$ \\
\hline 28 & wild-type & $5 / 5$ & ND** & $5 / 5$ & $5 / 5$ & $4 / 5$ \\
& Darp3 & $4 / 5$ & ND & $3 / 5$ & $3 / 5$ & $5 / 5$ \\
\hline 42 & wild-type & $3 / 3$ & $3 / 3$ & $3 / 3$ & $3 / 3$ & $3 / 3$ \\
& Darp3 & $3 / 5$ & $5 / 5$ & $4 / 5$ & $3 / 5$ & $2 / 5$ \\
\hline
\end{tabular}

* number positive/number tested.

** ND = not done.
SD flaB copy numbers, $P=0.0002)$. Tick cohorts from individual $\Delta$ arp3 infected mice contained 9/10, 5/10, 10/ $10,6 / 10$ and $10 / 10$ positive ticks. Results demonstrated that $\Delta$ arp3 can be acquired by ticks from infected $\mathrm{C} 3 \mathrm{H}$ mice, but ticks that acquired $\Delta$ arp3 harbored fewer organisms compared to wild-type.

The ability of $\Delta$ arp3 spirochetes to be transmitted from infected ticks to naïve $\mathrm{C} 3 \mathrm{H}$ mice was next evaluated by placing 10 nymphal ticks from the wild-type and $\Delta$ arp3 positive tick cohorts (above) onto each recipient mouse. Mice were necropsied at 3 weeks following tick feeding, and ear, heart base, ventricular muscle, tibiotarsus and quadriceps muscle were tested by flaB Q-PCR. Among 5 mice fed upon by ticks carrying wild-type spirochetes, 4/5 mice became infected, and all tissue sites from the 4 positive mice were PCR-positive, with high copy numbers of flaB DNA in tissues (Figure 3). In contrast, 2 of the 7 mice that were fed upon by $\Delta$ arp3 infected ticks were positive, but only a single tissue in each of the positive mice contained low copy numbers of $f l a B$ DNA. Results indicated that $\Delta$ arp3 spirochetes are capable of tick-borne transmission. Since ticks infected with $\Delta$ arp3 spirochetes had significantly fewer spirochete loads compared to ticks infected with wildtype spirochetes, it could not be concluded that there was less efficient transmission.

\section{Discussion}

This study examined the effect of targeted deletion of BBF01/arp on infectivity of B. burgdorferi B31. The median infectious dose of B. burgdorferi B31 with an arp null mutation was elevated approximately ten-fold compared to wild-type spirochetes, and restored by complementation. Therefore, it is apparent that BBF01/arp is not essential for infectivity of the mammalian host. This is supported by indirect results of others, who demonstrated diminished infectivity in B. burgdorferi spirochetes lacking linear plasmid 28-1 (lp28-1), which encodes only two unique and functional genes, $v l_{s} E$ and arp [25-29]. Furthermore, clones of B. burgdorferi B31 

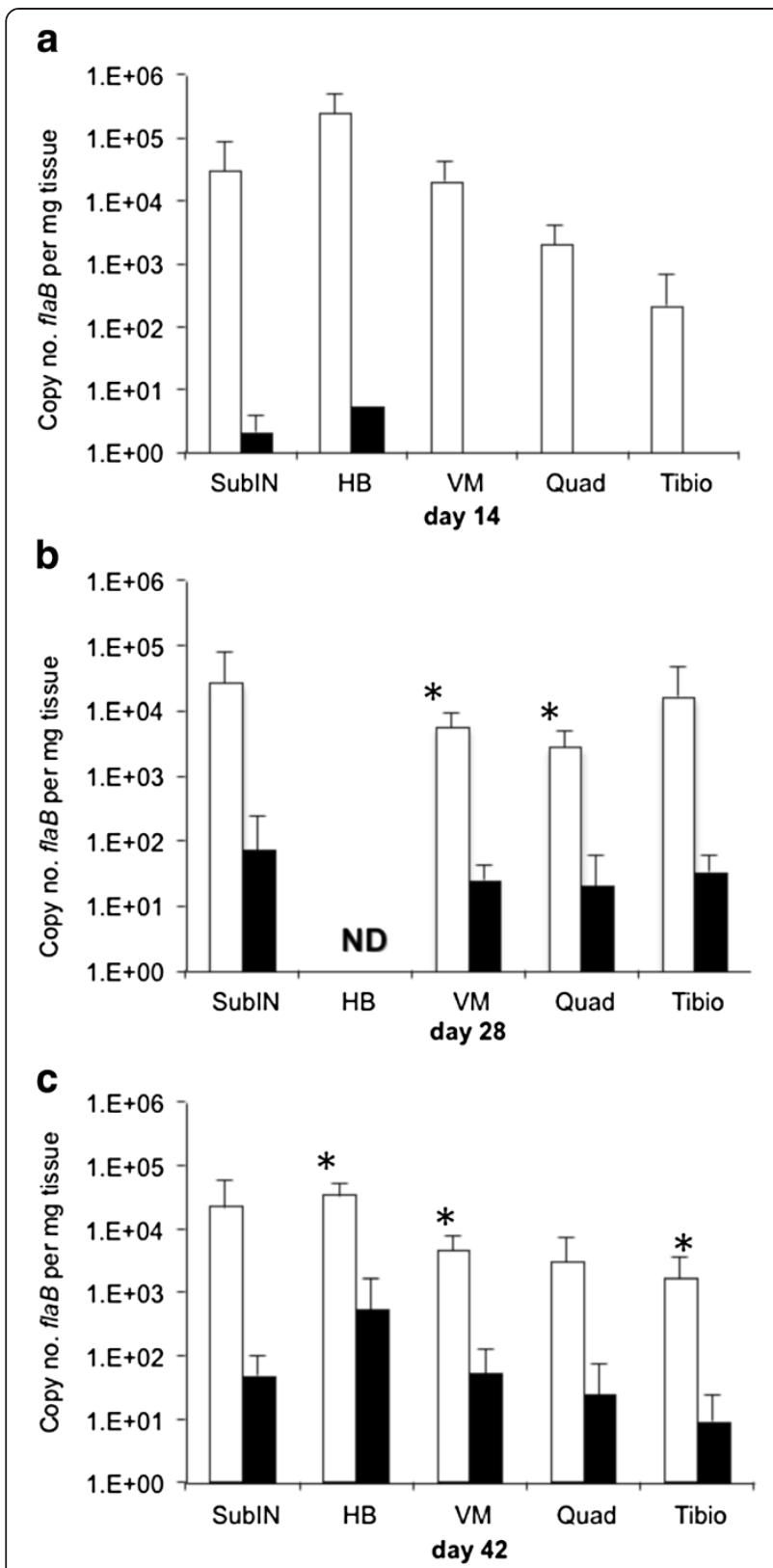

Figure 2 Borrelia burgdorferi flaB DNA copies per mg tissue weight (means \pm standard deviations) in PCR-positive tissues summarized in Tables 2 and 3, including sub-inoculation site (subIN), heart base (HB), ventricular muscle (VM), quadriceps muscle (Quad) and tibiotarsus (Tibio) from C3H mice inoculated with wild-type (white bars) compared to arp null $\Delta$ arp3 $B$. burgdorferi (black bars) at day 14 (a), day 28 (b) and day 42 (c) of infection. $(*, P \leq 0.05)$ ND: not determined.

with a deletion of the left side of lp28-1, which contains arp, remained infectious and capable of persistence, similar to wild-type spirochetes [25].

Examination of the pathogenicity of various $B$. burgdorferi B31 clones lacking lp28-1 has shown that clones lacking lp28-1 were infectious in BALB/c-scid

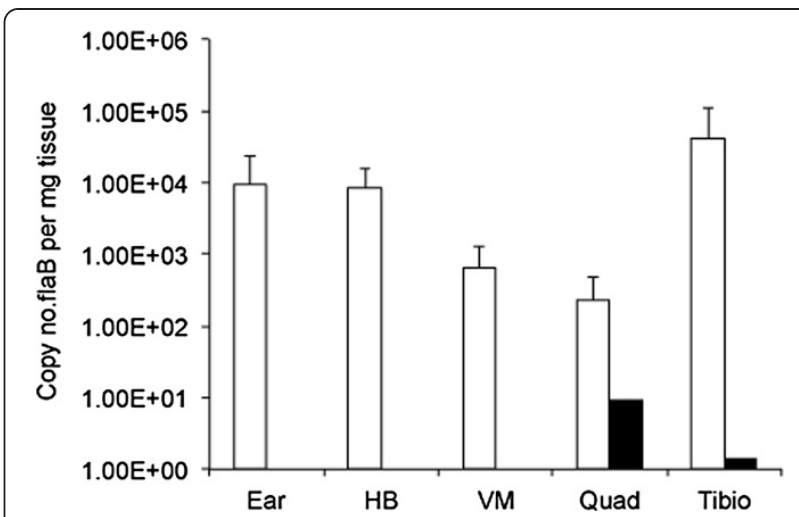

Figure 3 Borrelia burgdorferi flaB DNA copies per mg tissue weight (means \pm standard deviations) in PCR-positive tissues, including ear, heart base (HB), ventricular muscle (VM), quadriceps muscle (QM) and tibiotarsus (Tt) of mice at 3 weeks after feeding of nymphal ticks from tick cohorts infected with wild-type or arp null $\Delta \operatorname{arp} 3$ B. burgdoferi.

mice and reached similar tissue burdens as wild-type spirochetes, but were incapable of inducing arthritis [29]. Results of the current study indicate that infection of C3H-scid mice with arp null spirochetes resulted in lower spirochete burdens in tissues with development of equally severe arthritis as that induced by wild-type spirochetes. Thus, it appears that arp null spirochetes are equally (if not more) arthritogenic than wild-type $B$. burgdorferi in $\mathrm{C} 3 \mathrm{H}$-scid mice. The lack of effect on tissue burdens and arthritis in BALB/c-scid mice infected with $B$. burgdorferi devoid of the entire lp28-1 plasmid, but reduced burdens in infections with arp null spirochetes observed in the current study are likely due to the experimental variations in $B$. burgdorferi strains (B31-5A11 vs. B31-A3), mouse strains (BALB/c-scid vs. $\mathrm{C} 3 \mathrm{H}$-scid), or a number of other possible genetic variables.

Lack of lp28-1 has been associated with failure to persist in immunocompetent mice. This has been attributed to $v l s E$, since clones lacking a region of the plasmid that encodes arp are capable of persistent infection [25,29]. The current study examined persistence in immunocompetent $\mathrm{C} 3 \mathrm{H}$ mice up to 42 days after inoculation, and demonstrated that arp null spirochetes were indeed capable of persistence. In the present study, we also infected mice for antibody evaluation at 60 days of culture-confirmed infection, and thus verified persistence for up to 60 days. As in $\mathrm{C} 3 \mathrm{H}$-scid mice, arp null spirochete burdens were lower in $\mathrm{C} 3 \mathrm{H}$ mouse tissues compared to wild-type spirochetes. Notably, arthritis severity was markedly reduced in $\mathrm{C} 3 \mathrm{H}$ mice infected with arp null spirochetes. Since arp null spirochetes are fully arthritogenic in SCID mice, these results suggest that the lower pathogenicity of arp null spirochetes in immunocompetent mice is a consequence of susceptibility 
of the arp null mutant to immune response. Other antigens that are expressed during infection have also been shown to be susceptible to arthritis-resolving antibody responses, including DbpA [8], BmpA, and BmpB [30]. In the absence of Arp, these or other antigens may be targets of immune-mediated phenotypic effects noted in the present study.

Although arp null spirochetes are capable of surviving in the murine host, their ability to do so appears to be compromised, since arp null spirochete burdens were 2 logs fewer in tissues of SCID mice compared to wild type spirochetes, and were even lower in immunocompetent mice. Thus, arp null spirochetes appear to be either less fit to grow or are more vulnerable to innate and acquired immune factors compared to wild type spirochetes. This lack of fitness is likely responsible for the additional phenotypic effect of arp deletion that was observed in acquisition and transmission by vector ticks. Larval ticks were fed upon mice infected with wild-type or arp null spirochetes, and allowed to molt into nymphs. Ticks became infected with both types of spirochetes, but following molting, nymphal ticks that were colonized with arp null spirochetes had significantly lower spirochete loads per tick compared to ticks colonized with wild type spirochetes. The lower arp null spirochete loads were likely influenced by the lower spirochete burdens in tissues of mice that were fed upon by the ticks. This effect has been demonstrated by others [31] in which ticks that fed upon MyD88 deficient mice infected with $B$. burgdorferi had higher spirochete burdens compared to ticks that fed upon wild-type mice. MyD88 deficient mice have significantly higher spirochete tissue burdens compared to wild-type mice. The lower rate of transmission of arp null spirochetes from infected nymphal ticks to naïve mice could also have been influenced lower spirochete burdens in arp null colonized ticks. Further studies are needed to examine dynamics within ticks, but there is normally a significant burst of replication of spirochetes within fed ticks [32] that did not appear to occur in ticks colonized with arp null spirochetes. Nevertheless, results indicated that arp null spirochetes could be acquired and transmitted by vector ticks, albeit at diminished levels.

\section{Conclusion}

Deletion of the arp gene resulted in a modest phenotypic effect, including reduced infectious dose, reduced fitness of B. burgdorferi for growth in the mammalian host, and reduced ability for acquisition and transmission by the vector tick. Deletion of a number of $B$. burgdorferi genes has been found to have only mild phenotypic effects upon infectivity and persistence of $B$. burgdorferi (reviewed in [33]). This is likely due in large part to compensatory up-regulation of other genes.
Although the function of Arp remains unknown, the current study in which arp was deleted with relatively modest phenotypic effects underscores the complexity of B. burgdorferi biology and emphasizes caution in attributing phenotype or lack thereof to the role of a single gene alteration.

\section{Methods}

\section{Mice}

Specific-pathogen-free, 3 to 5 week old $\mathrm{C} 3 \mathrm{H} / \mathrm{HeN}(\mathrm{C} 3 \mathrm{H})$ and severe combined immunodeficient (SCID) $\mathrm{C} 3 \mathrm{H} /$ Smn.CIcrHsd-Prkdc scid (C3H-scid) mice were obtained from Frederick Cancer Research Center (Frederick, MD) and Harlan Sprague Dawley, Inc. (Indianapolis, IN), respectively. Pregnant Swiss outbred Crl:CD1(ICR) mice were obtained from Charles River Laboratories (Hollister, CA). Mice were infected by subdermal inoculation of mid-log phase B. burgdorferi in $0.1 \mathrm{ml}$ culture medium on the dorsal thoracic midline. Mice were killed by carbon dioxide narcosis and exsanguination by cardiocentesis. Infection status of mice was confirmed at necropsy by culture of the urinary bladder and subinoculation site, as described [4]. Animal use was approved by the University of California Davis Animal Care and Use Committee. University of California Davis has a Public Health Service Animal Welfare Assurance on file and is fully accredited by the Association for the Assessment and Accreditation of Laboratory Animal Care International.

\section{Histopathology}

Joint (knee and tibiotarsus) and heart tissues were fixed in neutral buffered formalin, demineralized, paraffinembedded, sectioned, and stained with hematoxylin and eosin. Tissues were blindly evaluated and tibiotarsal arthritis severity was scored on a scale of 0 (negative), 1 (mild), 2 (moderate) or 3 (severe). Based upon extensive use of this scoring system, a score of 3 is generally limited to SCID mice, and a score of 1-2 is typical of immunocompetent $\mathrm{C} 3 \mathrm{H}$ mice $[4,34,35]$. The prevalence of carditis was also blindly recorded, but a severity score is not possible with carditis, due to variation in severity among mice within a particular treatment group, thereby precluding accurate scoring [34].

\section{Bacterial strains}

Low passage infectious B. burgdorferi s.s. strain B31-A3 (wild-type) was acquired from D. Scott Samuels, University of Montana, and utilized as both a wild-type control and for genetic manipulation. B31-A3 is a clonal isolate of B31 MI, the prototype B31 strain utilized for genome sequencing [36,37]. An additional B31-A3 variant, $B$. burgdorferi B31-A3-lp28-1-G, containing a gentamicin resistance gene on lp28-1 [38], was provided by D. Scott 
Samuels (originally from P. Rosa, Rocky Mountain Laboratories). Spirochetes were grown in modified Barbour Stoenner Kelly (BSKII) medium [39] with 6\% rabbit serum. Inocula were enumerated by dark-field microscopy using a Petroff-Hausser chamber immediately prior to use, and serial 10-fold dilutions were prepared for evaluating median infectious doses. For isolation of transformants, spirochetes were cultured on semi-solid gelatin-free BSKII medium supplemented with $1.7 \%$ dissolved agarose plus appropriate antibiotic $(50 \mu \mathrm{g} / \mathrm{ml}$ streptomycin or $40 \mu \mathrm{g} / \mathrm{ml}$ gentamicin). Escherichia coli cloning strain TOP10F' (Invitrogen, Inc., CA), was grown in Luria-Bertani broth under aerobic conditions at $37^{\circ} \mathrm{C}$. Transformed E. coli were selectively cultured in broth medium with $50 \mu \mathrm{g} / \mathrm{ml}$ spectinomycin.

\section{Genetic modification of B. burgdorferi}

Arp null mutants ( $\Delta$ arp) were constructed by exchange of the arp open reading frame (ORF) with a mutagenic cassette via homologous recombination. The mutagenic cassette consisted of a streptomycin-spectinomycin resistance cassette, flaB-aadA (kindly provided by D. Scott Samuels, University of Montana, Missoula, MT), flanked by regions of the B. burgdorferi B31-A3 plasmid lp28-1 that flanked the arp gene at both the 5' and 3' regions. Single Overlap Extension PCR (SOEing) was used to join each part of the mutagenic cassette through primers containing overlapping homology (Table 4). First, the 5' flanking region (258bp) was amplified using primers ARP01 and the SOEing primer ARP02, which included homology to the $5^{\prime}$ region of the flaB-aadA PCR product. The flaB-aadA product (1199bp) was amplified using primers ARP03 and the SOEing primer ARP04, which included homology to the $5^{\prime}$ region of the $3^{\prime}$ region PCR product. The 3' flanking region (1309bp) was amplified using primers ARP05 and ARP06. Each part was gel purified using the Qiagen Gel Extraction Kit
(Qiagen Inc., Valencia, CA). SOEing was performed using a $2 \mu \mathrm{l}$ aliquot of each part mixed with $0.5 \mu \mathrm{l}(10$ $\mu \mathrm{M})$ each of primers ARP01 and ARP06 to produce a fragment that was 2766bp in length. The resulting mutagenic cassette was cloned into the $3.9 \mathrm{~kb}$ commercial vector, pCR2.1 TOPO (Invitrogen Corp., Carlsbad, CA) to produce a $7.5 \mathrm{~kb}$ suicide vector, "pKH-1". Plasmid DNA of pKH-1 (5-10 $\mu \mathrm{g})$ was electroporated into wildtype $B$. burgdorferi using the previously described protocol [40]. Transformants were selected by plating onto semi-solid BSKII medium (gelatin-free BSKII medium supplemented with $1.7 \%$ dissolved agarose and $50 \mu \mathrm{g} / \mathrm{ml}$ streptomycin). Clones that survived antibiotic selection were analyzed by PCR to confirm allele exchange using a combination of primers exterior and interior of the integration site (Table 4). PCR was performed to confirm the absence of the arp gene in several potential mutants. Plasmid profiling of $\Delta$ arp mutants was performed by PCR as previously described [28] to select mutants that contained important plasmids, including cp9 (rev), cp26 (ospC), ср32-1 (BBP33), ср32-2/7 (BBO32), ср32-3 (ospG), ср32-6 (BBM32), сp32-8 (BBL32-34), ср32-9 (BBN32-33), lp17 (BBD12-13), lp21 (BBU06-07), lp25 (pncA), lp28-1 ( $v l s E)$, lp28-3 (BBH17), lp28-4 (non-coding region), lp36 (BBK12), lp38 (ospD), lp54 (ospA), and lp56 (BBQ67), using previously published primers [28,41]. One of the $\Delta$ arp clones ( $\Delta$ arp3) that retained the same complete set of plasmids as the wild-type isolate was used in further experiments.

The $\Delta$ arp3 clone was complemented with a whole lp28-1 plasmid that contained the arp gene and a selection marker for gentamicin (lp28-1-G). This plasmid was knocked in to replace the endogenous lp28-1 (where arp was deleted), as previously published [38]. Plasmid DNA containing lp28-1-G was purified from B. burgdorferi B31-A3-lp28-1-G, electroporated into B31- $\Delta$ arp3 spirochetes, and then complemented transformants were

Table 4 Primers for construction of the arp mutagenic cassette and verification of allelic exchange

\begin{tabular}{|c|c|c|}
\hline Primer & Sequence $\left(5^{\prime}>3^{\prime}\right)$ & Application \\
\hline ARP01 & GCCTTCGTTAAGGTTTTGTT & amplify arp upstream homology \\
\hline \multirow[t]{2}{*}{ ARP02 } & GGAAATCTTCCTTGAAGCTCGGGTACAA & SOEing arp upstream homology \\
\hline & GTTGTTCCTCCTAAATTAAATAAAAATAA & to aadA cassette \\
\hline ARP03 & TACCCGAGCTTCAAGGAAG & amplify aadA cassette \\
\hline \multirow[t]{2}{*}{ ARP04 } & GGTATATGTAATTTCGACTITAAGTTAAAAAT & SOEing arp downstream \\
\hline & CCGATTGTTTCATTTGCCGACTACCTTGGT & homology to aadA cassett \\
\hline ARP05 & GAACAATCGGATIIITAACTTAAAGTCG & amplify arp dowsteam homology \\
\hline ARP06 & ACCCCAGTAACTCAATTTCTAATTG & amplify arp dowsteam homology \\
\hline ARP07 & TTCTTGATTAGGGTAAAAAATTCT & check integration at $5^{\prime}$ end \\
\hline ARP08 & GTCTTGTATTGTTGAACAAAACACTT & check integration at $3^{\prime}$ end \\
\hline ARP09 & GTTCCATATGAGGGAAGCG & check integration within aadA \\
\hline ARP10 & CCAAGCGATCTTCTTCTTGTC & check integration within aadA \\
\hline
\end{tabular}


selected with gentamicin. A series of PCRs using diagnostic primers (Table 1 ) were used to identify clones that had undergone successful plasmid exchange of lp28-1 arp::aadA with lp28-1G by confirming the presence of the arp operon. Plasmid profiling was performed and the complemented isolate B31- $\Delta$ arp3-2.2 ( $\Delta$ arp3-lp28-1-G) was used for further analysis.

\section{Quantitative PCR}

DNA was extracted from sub-inoculation site, heart base, ventricular muscle, quadriceps muscle and right tibiotarsus (integument removed), and samples were subjected to real-time Q-PCR. In addition to the site of inoculation, four additional sites were evaluated, based upon previous studies demonstrating that they all become consistently infected [22], but manifest different patterns of inflammation. Heart base is the site where carditis occurs, whereas cardiac ventricular muscle develops minimal or no inflammation [34]. In addition, the tibiotarsal joint typically develops arthritis, whereas the adjacent quadriceps femoris muscle develops minimal or no inflammation [42]. Quantification of gene copies was based upon copy number per mg of tissue weight, as previously described [22]. DNA was extracted from samples using the DNeasy tissue kit, according to the manufacturer's instructions for tissues or insects (QIAGEN, Valencia, CA). In addition, DNA from B. burgdorferi cultured from mouse tissues was extracted for verification of genetic status of isolates. Three oligonucleotides, two primers and a probe, for the B. burgdorferi flaB and the arp genes were used, as previously described [19].

\section{Serology}

Immune sera were generated in $\mathrm{C} 3 \mathrm{H}$ mice inoculated with $10^{5}$ wild-type, $\Delta$ arp3, or $\Delta$ arp $3+\operatorname{lp} 28-1 G$ spirochetes at 60 days of infection. Infection was verified by culture, and individual sera were tested by enzyme linked immunosorbent assay (ELISA) to verify the appropriate presence or absence of Arp-reactive antibody. Three-fold dilutions (starting at 1:300) of immune sera were titrated by ELISA for antibody to B. burgdorferi B31 lysates and recombinant Arp, as described [11]. Samples were tested in duplicate, and each assay included uninfected mouse serum as a negative control and wild-type infected mouse serum as a positive control.

\section{Tick acquisition and transmission}

Ixodes scapularis ticks were acquired from Durland Fish, Yale University, as a single cohort of larvae from a pathogen-free laboratory-reared colony. In order to determine the ability of ticks to acquire infection, 40 larval ticks were placed on each mouse infected with either wild-type or $\Delta$ arp3 spirochetes. Replete (fed) ticks were collected as cohorts from each mouse and allowed to harden and molt into nymphal ticks. Randomly selected ticks from each mouse/tick cohort were tested for flaB and $\operatorname{arp}$ by Q-PCR. Remaining nymphal ticks in each cohort were placed on naïve $\mathrm{C} 3 \mathrm{H}$ mice to assess the relative ability of infected nymphal ticks to transmit wild-type or $\Delta$ arp3 spirochetes.

\section{Statistical analysis}

Multiple comparison analyses were performed using independent samples $t$-test or one-way analysis of variance, followed by post-hoc pair-wise comparisons (Tukey's HSD test) (PASW Statistics v. 18.0). Calculated $P$ values $\leq 0.05$ were considered significant. The median infectious dose $\left(\mathrm{ID}_{50}\right)$ was calculated using the method of Reed and Muench [43].

\section{Competing interests}

The authors declared that they have no competing interests.

\section{Authors' contributions}

$\mathrm{DI}, \mathrm{KH}, \mathrm{EH}$ and SWB performed and analyzed results. SF, EH and SWB participated in experimental design. DI, KH, EH and SWB co-wrote the manuscript. All authors read and approved the manuscript.

\section{Acknowledgments}

The generous technical guidance of D. Scott Samuels is particularly appreciated, and we are also grateful for the provision of ticks by Durland Fish. This work was supported by NIH/NIAID grants R01 Al-26815 and T32 Al-060555 (DI), and by NIH/ORIP T32 OD-011147 (DI).

Received: 7 January 2013 Accepted: 16 April 2013 Published: 7 May 2013

\section{References}

1. Steere AC, Schoen RT, Taylor E: The clinical evolution of Lyme arthritis. Ann Intern Med 1987, 107:725-731.

2. Steere AC, Coburn J, Glickstein L: The emergence of Lyme disease. J Clin Invest 2004, 113:1093-1101.

3. Steere AC: Lyme disease. N Engl J Med 1989, 321:586-596.

4. Barthold SW, de Souza MS, Janotka JL, Smith AL, Persing DH: Chronic Lyme borreliosis in the laboratory mouse. Am J Pathol 1993, 143(3):951-971.

5. McKisic MD, Barthold SW: T-cell-independent responses to Borrelia burgdorferi are critical for protective immunity and resolution of Lyme disease. Infect Immun 2000, 68:5190-5197.

6. Barthold SW, de Souza M, Feng S: Serum-mediated resolution of Lyme arthritis in mice. Lab Invest 1996, 74:57-67.

7. Barthold SW, Feng S, Bockenstedt LK, Fikrig E, Feen K: Protective and arthritis- resolving activity in serum from mice infected with Borrelia burgdorferi. Clin Infect Dis 1997, 25:S9-S17.

8. Barthold SW, Hodzic E, Tunev S, Feng S: Antibody-mediated disease remission in the mouse model of Lyme borreliosis. Infect Immun 2006, 74:4817-4825

9. de Silva AM, Fikrig E: Borrelia burgdorferi genes selectively expressed in ticks and mammals. Parasit Today 1997, 13:267-270.

10. de Silva AM, Fikrig E: Arthropod- and host-specific gene expression by Borrelia burgdorferi. J Clin Invest 1997, 99:377-379.

11. Feng S, Hodzic E, Stevenson B, Barthold SW: Humoral immunity to Borrelia burgdorferi $\mathrm{N} 40$ decorin binding proteins during infection of laboratory mice. Infect Immun 1998, 66:2827-2835

12. Feng $\mathrm{S}$, Hodzic $E$, Barthold SW: Lyme arthritis resolution with antiserum to a 37- kilodalton Borrelia burgdorferi protein. Infect Immun 2000, 68:4169-4173.

13. Fikrig E, Chen M, Barthold SW, Anguita J, Feng W, TelfordIII SR, Flavell RA Borrelia burgdorferi erpT expression in the arthropod vector and murine host. Mol Microbiol 1999, 31:281-290. 
14. Hanson MS, Cassatt DR, Guo BP, Patel NK, McCarthy MP, Dorward DW, Hook M: Active and passive immunity against Borrelia burgdorferi decorin binding protein A (DbpA) protects against infection. Infect Immun 1998, 66:2143-2153.

15. Hagman KE, Yang X, Wikel SK, Schoeler GB, Caimano MJ, Radolph JD, Norgard MV: Decorin-binding protein A (DbpA) of Borrelia burgdorferi is not protective when immunized mice are challenged via tick infestation and correlates with the lack of DbpA expression by B. burgdorferi in ticks. Infect Immun 2000, 68:4759-4764.

16. Feng S, Hodzic E, Freet K, Barthold SW: Immunogenicity of Borrelia burgdorferi arthritis-related protein. Infect Immun 2003, 71:7211-7214.

17. Liang FT, Jacobs MB, Bowers LC, Philipp MT: An immune evasion mechanism for spirochetal persistence in Lyme borreliosis. J Exp Med 2002, 195:415-422

18. Zhong W, Gern L, Stehle T, Museteanu C, Kramer M, Wallich R, Simon MM: Resolution of experimental and tick-borne Borrelia burgdorferi infection in mice by passive, but not active immunization using recombinant OspC. Eur J Immunol 1999, 29:946-957.

19. Hodzic E, Feng S, Freet KJ, Borjesson DL, Barthold SW: Borrelia burgdorferi population kinetics and selected gene expression at the host-vector interface. Infect Immun 2002, 70:3382-3388.

20. Salazar CA, Rothemich M, Drouin EE, Glickstein L, Steere AC: Human Lyme arthritis and the immunoglobulin $\mathrm{G}$ antibody response to the 37-kilodalton arthritis-related protein of Borrelia burgdorferi. Infect Immun 2005, 73:2951-2957.

21. Tunev SS, Hastey CJ, Hodzic E, Feng S, Barthold SW, Baumgarth N: Lymphadenopathy during Lyme borreliosis is caused by spirochete migration- induced specific B cell activation. PLoS Pathog 2011, 7:e1002066.

22. Hodzic E, Feng S, Freet K, Barthold SW: Borrelia burgdorferi population dynamics and prototype gene expression during infection of immunocompetent and immunodeficient mice. Infect Immun 2003, 71:5042-5055

23. Liang FT, Yan J, Mbow ML, Sviat SL, Gilmore RD, Mamula M, Fikrig E: Borrelia burgdorferi changes its surface antigenic expression in response to host immune responses. Infect Immun 2004, 72:5759-5767.

24. Probert WS, LeFebvre RB: Protection of $\mathrm{C} 3 \mathrm{H} / \mathrm{HeN}$ mice from challenge with Borrelia burgdorferi through active immunization with OspA, OspB, or OspC, but not with OspD or the 83-kilodalton antigen. Infect Immun 1994, 62:1920-1926.

25. Bankhead T, Chaconas G: The role of VlsE antigenic variation in the Lyme disease spirochete: persistence through a mechanism that differs from other pathogens. Mol Microbiol 2007, 65:1547-1558.

26. Labandeira-Rey M, Seshu J, Skare J: The absence of linear plasmid 25 or 28-1 of Borrelia burgdorferi dramatically alters the kinetics of experimental infection via distinct mechanisms. Infect Immun 2003, 71:4608-4613.

27. Labandeira-Rey M, Skare JT: Decreased infectivity in Borrelia burgdorferi strain B31 is associated with loss of linear plasmid 25 or 28-1. Infect Immun 2001, 69:446-455.

28. Purser JE, Norris SJ: Correlation between plasmid content and infectivity of Borrelia burgdorferi. Proc Natl Acad Sci USA 2000, 97:13865-13870.

29. Xu Q, Seemanapalli SV, Lomax L, McShan K, Li X, Fikrig E, Liang FT: Association of linear plasmid 28-1 with an arthritic phenotype of Borrelia burgdorferi. Infect Immun 2005, 73:7208-7215.

30. Pal U, Wang P, Bao F, Yang X, Samanta S, Schoen R, Wormser GP, Schwartz I, Fikrig E: Borrelia burgdorferi basic membrane proteins $A$ and $B$ participate in the genesis of Lyme arthritis. J Exp Med 2008, 205:133-141.

31. Bockenstedt LK, Liu N, Schwartz I, Fish D: MyD88 deficiency enhances acquisition and transmission of Borrelia burgdorferi by Ixodes scapularis ticks. Infect Immun 2006, 74:2154-2160.

32. Hodzic E, Borjesson DL, Feng S, Barthold SW: Acquisition dynamics of Borrelia burgdorferi and the agent of human granulocytic ehrlichiosis at the host-agent interface. Vector Borne Zoonotic Dis 2001, 1:149-158.

33. Kung F, Anguita J, Pal U: Borrelia burgdorferi and tick proteins supporting pathogen persistence in the vector. Future Microbiol 2013, 8:41-56.

34. Armstrong AL, Barthold SW, Persing DH, Beck DS: Carditis in Lyme disease susceptible and resistant strains of laboratory mice infected with Borrelia burgdorferi. Am J Trop Med Hyg 1992, 47(2):249-258.
35. Barthold SW, Sidman CL, Smith AL: Lyme borreliosis in genetically resistant and susceptible mice with severe combined immunodeficiency. Am J Trop Med Hyg 1992, 47:605-613.

36. Casjens S, Palmer N, van Vugt R, Huang WM, Stevenson B, Rosa P, Lathigra $R$, Sutton G, Peterson J, Dodson RJ, et al: A bacterial genome in flux: the twelve linear and nine circular extrachromosomal DNAs in an infectious isolate of the Lyme disease spirochete Borrelia burgdorferi. Mol Microbiol 2000, 35:490-516.

37. Fraser CM, Casjens S, Huang WM, Sutton GG, Clayton R, Lathigra R, White $O$, Ketchum KA, Dodson R, Hickey EK, et al: Genomic sequence of a Lyme disease spirochaete, Borrelia burgdorferi. Nature 1997, 390:580-586.

38. Grimm D, Eggers CH, Caimano MJ, Tilly K, Stewart PE, Elias AF, Radolf JD, Rosa PA: Experimental assessment of the roles of linear plasmids Ip25 and Ip28-1 of Borrelia burgdorferi throughout the infectious cycle. Infect Immun 2004, 72:5938-5946.

39. Barbour AG: Isolation and cultivation of Lyme disease spirochetes. Yale J Biol Med 1984, 57:521-525.

40. Samuels DS, Mach KE, Garon CF: Genetic transformation of the Lyme disease agent Borrelia burgdorferi with coumarin-resistant gyrB. J Bacteriol 1994, 176:6045-6049.

41. Ohnishi J, Piesman J, de Silva A: Antigenic and genetic heterogeneity of Borrelia burgdorferi populations transmitted by ticks. Proc Natl Acad SC USA 2001, 98:670-675.

42. Barthold SW, Persing DH, Armstrong AL, Peeples RA: Kinetics of Borrelia burgdorferi dissemination and evolution of disease following intradermal inoculation of mice. Am J Pathol 1991, 139:263-273.

43. Reed $\sqcup$, Muench $H$ : A simple method of estimating fifty per cent endpoints. Am J Hyg 1938, 27:493-497.

doi:10.1186/1471-2180-13-100

Cite this article as: Imai et al.: Influence of arthritis-related protein (BBF01) on infectivity of Borrelia burgdorferi B31. BMC Microbiology 2013 $13: 100$

\section{Submit your next manuscript to BioMed Central and take full advantage of:}

- Convenient online submission

- Thorough peer review

- No space constraints or color figure charges

- Immediate publication on acceptance

- Inclusion in PubMed, CAS, Scopus and Google Scholar

- Research which is freely available for redistribution

Submit your manuscript at www.biomedcentral.com/submit
C Biomed Central 